\title{
Perforating Peripheral Ulcerative Keratitis in Syphilis
}

\author{
Pimpiroon Ploysangam Ruth Marie Mattern \\ Department of Ophthalmology, Ross Eye Institute, State University of New York at \\ Buffalo, Buffalo, NY, USA
}

\section{Keywords}

Corneal perforation · Syphilis · Peripheral ulcerative keratitis · Pustular rash · Human immunodeficiency virus

\begin{abstract}
Purpose: To present a case of peripheral ulcerative keratitis with perforation in the setting of syphilis. Method: A single observational case report in urban USA. Case Presentation: A patient with multiple sexual partners presented with bilateral peripheral ulcerative keratitis, unilateral corneal perforation, and a pustular rash. Workup was positive for syphilis as well as HSV, HIV, and multiple autoantibody tests. Findings improved after treatment for syphilis. Conclusion: Bilateral peripheral ulcerative keratitis in syphilis has been reported in one prior case. However, corneal perforation in the setting of syphilis infection has not, to our knowledge, been reported previously.

(C) 2019 The Author(s) Published by S. Karger AG, Basel
\end{abstract}

\section{Introduction}

Peripheral ulcerative keratitis (PUK) involves crescent-shaped corneal ulcers adjacent to the limbus. Epithelial defects may occur over areas of corneal stromal thinning, and adjacent scleral and conjunctival involvement may occur. Although areas of corneal stromal thinning are typically unilateral and sectoral, they may be bilateral and diffuse. Most cases are 


\section{Case Reports in Ophthalmology}

associated with autoimmune diseases; a small portion has been associated with infectious processes and vitamin A deficiency [1].

\section{Patient Case}

A 33-year-old African-American male presented with a 1-month history of bilateral eye pain, redness, and blurry vision. He had been seen by another provider and started on topical antibiotics for presumed conjunctivitis. His eye condition worsened, and he presented to our service with a complaint of throbbing pain in both eyes. On examination, he had a pustular rash all over his face, neck, and upper chest. Visual acuity was 20/100 uncorrected - pinhole $20 / 70$ in the right eye, and 20/150 uncorrected - pinhole 20/60 in the left eye. Multiple perilimbal stromal infiltrates were noted in both eyes, some with vascularization; thinning was noted superiorly and inferiorly. An inferotemporal perilimbal ulcer was noted in the right eye (Fig. 1). An inferotemporal perilimbal corneal perforation measuring $4.5 \mathrm{~mm}$ horizontally by $3 \mathrm{~mm}$ vertically plugged with prolapsed iris, as well as peripheral cornea thinning superotemporally, were noted in the left eye (Fig. 2). No scleral involvement was seen, although he did have significant conjunctival injection. Keratitic precipitates were documented bilaterally. The view of the posterior segment was very poor then, although the follow-up dilated fundus examination was unremarkable. Review of systems was negative for fevers, night sweats, weight loss, shortness of breath, coughs, myalgia, arthritis, history of severe acne, history of intravenous drug use, or travel history. The patient did admit to having unprotected sex with multiple female partners, but he denied any penile discharge, lesions, or oral ulcers. He had a history of chicken pox but denied a history of cold sores. At this point, hospital admission for additional workup was recommended, along with repair of the perforation. The patient refused repair at the time and was restarted on topical antibiotics. The patient was lost to followup at this point but re-presented 1 week later after no improvement. The patient was admitted and started on intravenous methylprednisolone $1,000 \mathrm{~g} /$ day.

Additional workup was obtained for autoimmune and infectious causes of the bilateral PUK. Labs were positive for antinuclear antibodies (anti-ANA) (titer of 1:1,280), anti-doublestranded deoxyribonucleic acid (anti-dsDNA) (131 units/mL; normal 0-99, indeterminate 100-120), anticentromere antibodies, antihistone antibodies, erythrocyte sedimentation rate (ESR) $(131 \mathrm{~mm} / \mathrm{h}$; normal 0-15), C-reactive protein (CRP) (48.62; normal 0.20-10.0), and lysozyme $(14.7 \mu \mathrm{g} / \mathrm{mL}$; normal 3.0-12.8). Lab tests were negative for perinuclear anti-neutrophil cytoplasmic antibodies (p-ANCA), cytoplasmic anti-neutrophil cytoplasmic antibodies (c-ANCA), atypical p-ANCA, Smith antibody, anti-Ro antibodies, anti-La antibodies, anti-ribonucleoprotein (RNP) antibodies, anti-topoisomerase I antibodies (Sc1-70), and elevated angiotensin-converting enzyme. Infectious serologies were also positive for human immunodeficiency virus (HIV-1) (viral load 118,589 RNA copies/mL), cluster of differentiation 4 (CD4) count $(462 / \mu \mathrm{L}$; normal $688-1,346)$, cluster of differentiation 8 (CD8 count) $(1,601 / \mu \mathrm{L}$; normal 243-762), herpes simplex virus II (HSV II) immunoglobulin G (IgG) (12.59), varicella zoster virus (VZV) IgG (1.28), hepatitis A, syphilis IgG, rapid plasma regain (RPR) $(1: 1,024)$, and cytomegalovirus (CMV) IgG (5.32). The patient was negative for tuberculosis, toxoplasmosis, hepatitis C, Bartonella henselae, Bartonella quintana, Lyme, Chlamydia trachomatis, and gonorrhea tests. Lumbar puncture showed a mild pleocytosis without any microbial growth. Corneal scrapings were negative for HSV polymerase chain reaction (PCR), and a culture of his right cornea grew Staphylococcus aureus; corneal scrapings were not checked for syphilis. Vitamin A level was normal, and toxicology urine screen was negative. 
With the positive HIV test, the methylprednisolone was discontinued after two doses. He was started on ganciclovir ( $5 \mathrm{mg} / \mathrm{kg}$ every $12 \mathrm{~h}$ ), but when the syphilis testing came back positive, the ganciclovir was discontinued after a 5-day course, and a 14-day course of intravenous penicillin $\mathrm{G}$ ( 4 million units every $4 \mathrm{~h}$ ) was administered.

\section{Results}

The patient agreed to a left corneal patch graft with temporary tarsorrhaphy, performed 1 week after admission. With syphilis treatment, the patient had significant visual improvement to 20/20 in both eyes. He was discharged home after completing the 2-week course of intravenous penicillin, with instructions to follow up in 1 week at the eye clinic and HIV clinic. When he returned 4 months later for a late follow-up, he had a visual acuity of 20/20 uncorrected in the right eye, and 20/30 uncorrected - pinhole 20/20 in the left eye. Only multiple perilimbal scars without thinning remained bilaterally, and the corneal patch graft was intact (see Fig. 3, 4). His pustular rash had also resolved. His temporary tarsorrhaphy and a loose suture in the patch graft were removed. He missed an additional appointment but was seen by ophthalmology after 7 months (1 year after initial presentation). He also had multiple visits at the HIV clinic to prescribe and monitor HIV treatment.

\section{Discussion}

Ocular syphilis most commonly manifests as uveitis and retinitis. Rare cases with corneal involvement have included stromal keratitis without ulcerations. However, Vignesh et al. [2] described a rare presentation of ocular syphilis as bilateral PUK in a patient in India whose bloodwork was positive for Venereal Disease Research Laboratory test (VDRL) and fluorescent treponemal antibody absorption (FTA-ABS). The patient's PUK improved with intravenous penicillin and topical steroids. Additionally, pustular rash as an extremely rare presentation of secondary syphilis has been reported. Pustular lesions have been noted in patients typically with poor health or with HIV. Variants of this include malignant lues seen in HIVpositive patients with syphilis and an acneiform variant localizing to the face and resembling acne vulgaris [3]. Our patient's positive RPR and syphilis IgG, mild pleocytosis in his cerebral spinal fluid, and dramatic improvement with syphilis treatment makes ocular syphilis the most likely primary diagnosis.

Other causes of PUK that may be considered for this patient include a disseminated herpetic infection leading to bilateral HSV epithelial keratitis or bilateral PUK. A case report by Praidou et al. [4] describes a patient with bilateral herpes simplex keratitis initially misdiagnosed as rheumatoid arthritis-related PUK; the correct diagnosis was made after corneal scrapings came back positive for HSV PCR and subsequent improvement after stopping topical prednisolone and adding topical acyclovir and systemic valacyclovir. Another case series by Neves et al. [5] describes 3 patients with acquired immunodeficiency syndrome (AIDS) presenting with PUK secondary to herpes zoster, treated with systemic antivirals; all 3 had skin involvement. Our patient's corneal scrapings were negative for HSV and VZV, and he significantly improved before receiving treatment for his herpetic infection, making this diagnosis less likely as the primary cause of his perforating keratitis.

Our patient had an elevated ESR and CRP and was positive for multiple autoantibody tests. ESR and CRP are nonspecific indicators of inflammation and would be expected to be 
elevated in a patient with any infection. ANA tests can be falsely positive in HIV [6]. AntidsDNA antibodies, anticentromere antibodies, and antihistone antibodies are overall highly specific for systemic lupus erythematosus, limited cutaneous systemic sclerosis, and drug-induced lupus, respectively [7-9]. However, this patient did not manifest any other signs of those autoimmune conditions. Furthermore, with full syphilis treatment and only two doses of methylprednisolone, his eye condition resolved without recurrence. Studies by ZandmanGoddard et al. [10] and Iordache et al. [11] have indicated that autoantibodies can be common and diverse in patients with HIV even without evidence of actual autoimmune disease. The positive autoantibody testing in this patient does not suffice to show that an underlying autoimmune disease, instead of syphilis, was the primary culprit in this PUK with perforation.

Though syphilis is arguably the primary cause of this patient's PUK, we cannot rule out the possibility that immune mechanisms relating to conditions other than syphilis played a contributing role in the development of severe PUK in this patient. The patient had multiple co-infections in addition to syphilis and HSV, including HIV (with a significant HIV viral load greater than $118,000 / \mathrm{mL}$ ) and CMV. HIV disease is recognized as a cause of dysregulation of the immune system $[10,11]$. Multiple infections and HIV-related immune aberration in this patient could have jointly acted to initiate the cascade of immune response that led to corneal melt and perforation.

In conclusion, the present case is, to our knowledge, the first reported case of perforating PUK in the setting of syphilis infection. A broad differential should be entertained for PUK with corneal perforation. Greater understanding of the complex immune response in syphilis and other co-infections may shed light on the mechanisms of corneal melt and perforation in cases such as the present one.

\section{Statement of Ethics}

The patient gave his written informed consent to publish his case (including the publication of images).

\section{Disclosure Statement}

The authors have no conflicts of interest to declare.

\section{Funding Sources}

No funding was received for this paper.

\section{Author Contributions}

Both authors contributed to the design and interpretation of the work, drafted and revised the work critically for important intellectual content, approved the final version to be published, and agreed to be accountable for all aspects of the work. 


\section{Case Reports in Ophthalmology}

\section{References}

1 Foster CS. Ocular manifestations of the potentially lethal rheumatologic and vasculitic disorders. J Fr Ophtalmol. 2013 Jun;36(6):526-32.

2 Vignesh AP, Srinivasan R, Vijitha S. Ocular syphilis masquerading as bilateral peripheral ulcerative keratitis. Taiwan J Ophthalmol. 2016 Oct-Dec;6(4):204-5.

3 Kazlouskaya V, Wittmann C, Tsikhanouskaya I. Pustular secondary syphilis: report of three cases and review of the literature. Int J Dermatol. 2014 Oct;53(10):e428-31.

4 Praidou A, Androudi S, Kanonidou E, Konidaris V, Alexandridis A, Brazitikos P. Bilateral herpes simplex keratitis presenting as peripheral ulcerative keratitis. Cornea. 2012 May;31(5):570-1.

5 Neves RA, Rodriguez A, Power WJ, Muccioli C, Lane L, Belfort R Jr, et al. Herpes zoster peripheral ulcerative keratitis in patients with the acquired immunodeficiency syndrome. Cornea. 1996 Sep;15(5):446-50.

6 Lane SK, Gravel JW Jr. Clinical utility of common serum rheumatologic tests. Am Fam Physician. 2002 Mar;65(6):1073-80.

7 Attar SM, Koshak EA. Medical conditions associated with a positive anti-double-stranded deoxyribonucleic acid. Saudi Med J. 2010 Jul;31(7):781-7.

8 Habash-Bseiso DE, Yale SH, Glurich I, Goldberg JW. Serologic testing in connective tissue diseases. Clin Med Res. 2005 Aug;3(3):190-3.

9 Kuramoto N, Ohmura K, Ikari K, Yano K, Furu M, Yamakawa N, et al. Anti-centromere antibody exhibits specific distribution levels among anti-nuclear antibodies and may characterize a distinct subset in rheumatoid arthritis. Sci Rep. 2017 Jul;7(1):6911.

10 Zandman-Goddard G, Shoenfeld Y. HIV and autoimmunity. Autoimmun Rev. 2002 Dec;1(6):329-37.

11 Iordache L, Bengoufa D, Taulera O, Rami A, Lascoux-Combe C, Day N, et al. Nonorgan-specific autoantibodies in HIV-infected patients in the HAART era. Medicine (Baltimore). 2017 Mar;96(10):e6230.

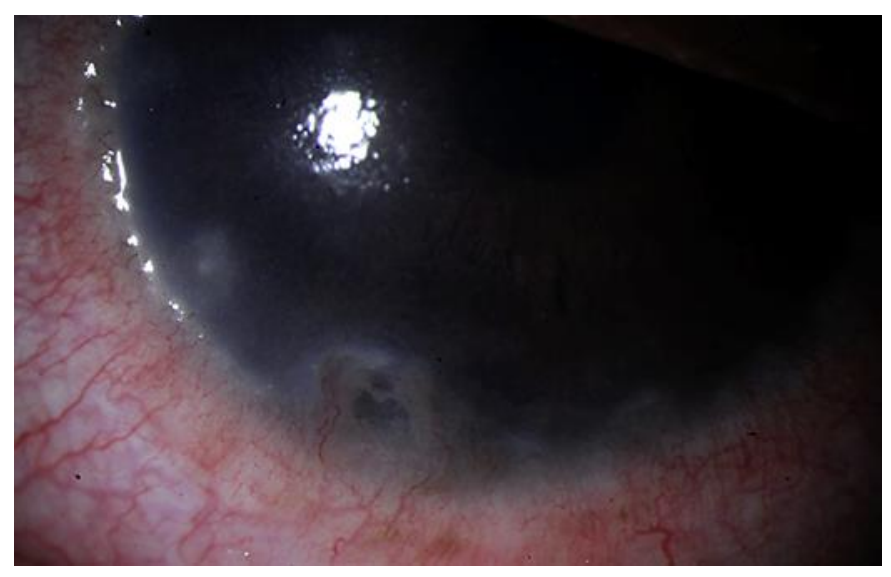

Fig. 1. External photo of the right eye at presentation. 


\section{Case Reports in Ophthalmology} www.karger.com/cop

Ploysangam and Mattern: Perforating Peripheral Ulcerative Keratitis in Syphilis

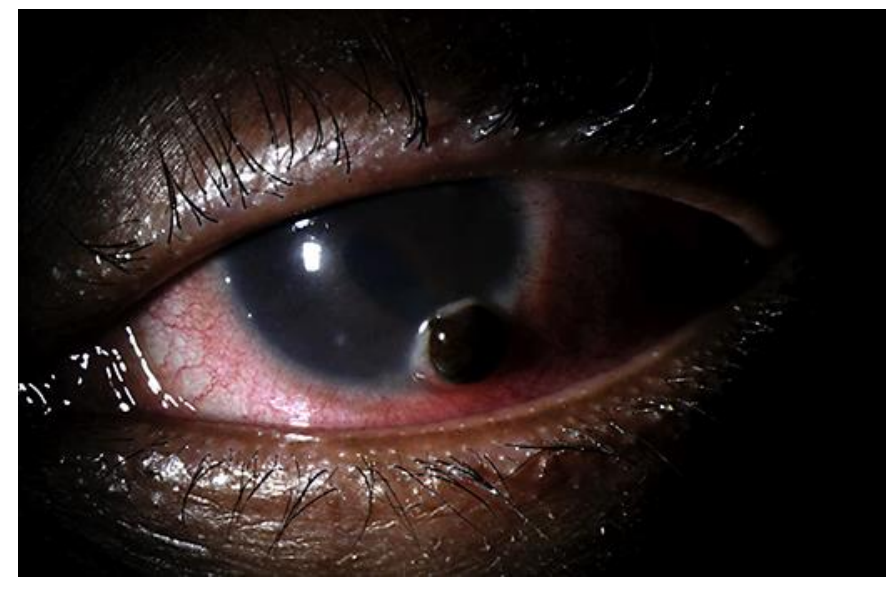

Fig. 2. External photo of the left eye at presentation.
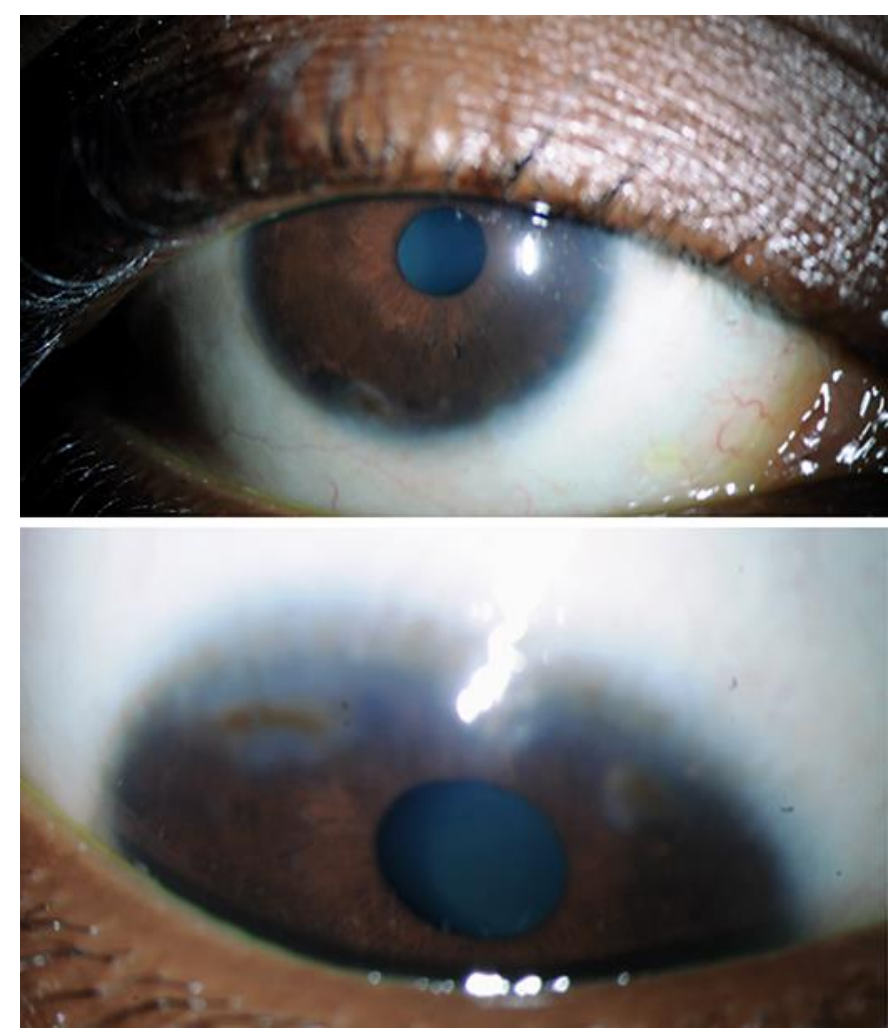

Fig. 3. External photos of the right eye at 4-month follow-up. 


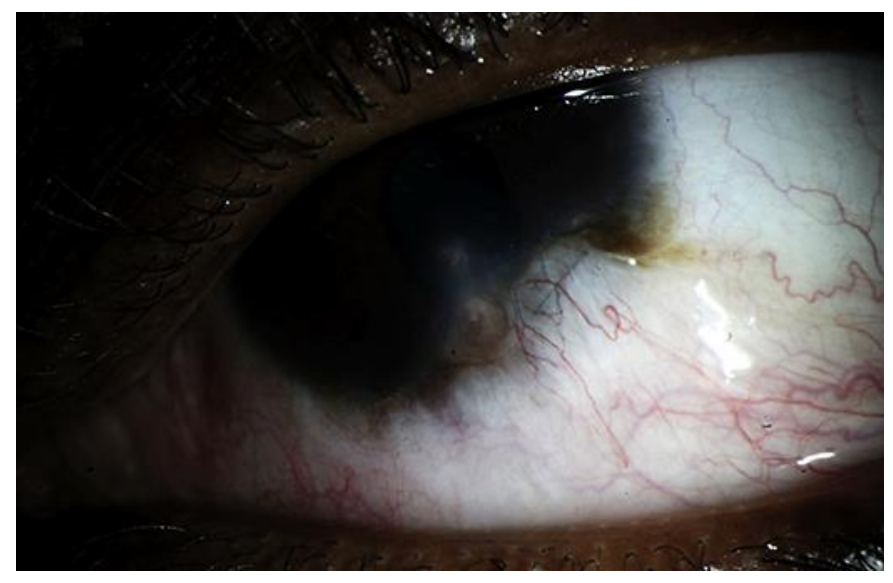

Fig. 4. External photo of the left eye at 4-month follow-up. 